\title{
Biofuel Powered Glucose Detection in Bodily Fluids with an n-type Conjugated Polymer
}

David Ohayon, ${ }^{1 \dagger}$ Georgios Nikiforidis, ${ }^{1 \dagger}$ Achilleas Savva, ${ }^{1}$ Andrea Giugni, ${ }^{2}$ Shofarul Wustoni, ${ }^{1}$ Tamilarasan Palanisamy, ${ }^{2}$ Xingxing Chen, ${ }^{2}$ Iuliana Petruta Maria, ${ }^{3}$ Enzo Di Fabrizio, ${ }^{2}$ Pedro M. F. J. Costa, ${ }^{2}$ Iain McCulloch, ${ }^{2,3}$ Sahika Inal ${ }^{1 *}$

${ }^{1}$ Biological and Environmental Science and Engineering Division, King Abdullah University of Science and Technology (KAUST), Thuwal 23955 - 6900, Saudi Arabia.

${ }^{2}$ Physical Science and Engineering Division, KAUST, Thuwal 23955-6900, Saudi Arabia.

${ }^{3}$ Department of Chemistry and Centre for Plastic Electronics, Imperial College London, London SW7 2AZ, United Kingdom.

$\dagger$ These authors contributed equally to this work.

*Corresponding author: sahika.inal@kaust.edu.sa

\begin{abstract}
N-type semiconducting polymers are a promising class of materials for applications relying on electron transfer for signal generation. Here we demonstrate the integration of an n-type conjugated polymer with a redox enzyme for autonomous detection of glucose and power generation from bodily fluids. The reversible, mediator-free, miniaturized glucose sensor is an enzyme coupled organic electrochemical transistor (OECT) with a detection range of six orders of magnitude. This n-type polymer is also used as anode and paired with a polymeric cathode in an enzymatic fuel cell to convert the chemical energy of glucose and oxygen into electrical power. The all-polymer biofuel cell shows performance scaling with the glucose content in solution and stability exceeding 30 days. Moreover, at physiologically relevant glucose concentrations and from
\end{abstract}


fluids such as human saliva, it generates enough power to operate an OECT, thus contributing to the technological advancement of self-powered micron-scale sensors and actutators that run on metabolites produced in the body. 
Biosensors contribute significant value to the healthcare industry, estimated at US\$13 billion annually, with glucose sensors representing $85 \%$ of the total market. ${ }^{1-3}$ As the primary source of energy in the human body, glucose performs various cell functions such as conduction of neurons, active transport, and synthesis of biochemical compounds. Any abnormality in glucose levels or its regulation leads to severe health conditions, as evidenced by diseases such as diabetes. Therefore, continuous monitoring of glucose levels is paramount for early diagnosis of diabetes as it is on the verge of becoming a pandemic disorder.

Today, the majority of electrochemical glucose sensors use enzymes as the recognition unit, especially glucose oxidase (GOx), because of their high selectivity and excellent catalytic activity towards glucose. ${ }^{4,5} \mathrm{GOx}$ electrochemically transforms glucose while being regenerated by ambient oxygen $\left(\mathrm{O}_{2}\right)$. The side product of this enzymatic reaction is hydrogen peroxide $\left(\mathrm{H}_{2} \mathrm{O}_{2}\right)$, which interacts with the transducer to generate an electrical signal that is proportional to glucose concentration. To improve selectivity and avoid $\mathrm{O}_{2}$ dependence as well as the co-oxidation of numerous species that can be oxidized at the relatively high potentials required for $\mathrm{H}_{2} \mathrm{O}_{2}$ electrooxidation, alternative detection strategies have been proposed. Modern sensors are designed such that they bypass $\mathrm{H}_{2} \mathrm{O}_{2}$ production. They employ either an artificial electron acceptor instead of $\mathrm{O}_{2}$ to mediate GOx cycling or an electronic transducer that electronically wires the enzyme to its surface, thus enabling electron transfer. ${ }^{6-8}$ Typical device configurations include passive electrodes, chemiresistors, and (water gated) field effect transistors as the transducer. ${ }^{9-11}$ However, these configurations possess several limitations. The low-amplitude biological signals hinder miniaturization of electronics due to the inverse scaling of electrode impedance with size, lowering the signal-to-noise ratio. For miniaturized platforms, on the other hand, the necessity to amplify these weak biological signals complicates sensor circuitry. Furthermore, all sensors, especially 
point-of-care devices, need a portable power supply or the capacity to recharge and stable electricity.

Oxidation of metabolites is one of the key processes that occur in our cells harvesting chemical energy to power cellular activity. Metabolites such as glucose or lactate are endogenous substances that are readily available in biological fluids and can be continuously renewed by metabolism, which makes them ideal fuels for powering bioelectronic devices. Enzymatic fuel cells (EFCs) that convert the energy of metabolism into electrical energy via biological pathways are one such example. ${ }^{12}$ The technological advancement of EFCs is ascribed mainly to the development of new electronic materials such as conjugated polymers, graphene, carbon nanotubes (CNTs) and metal nanoparticles. ${ }^{13}$ Yet, the relatively low power output of EFCs resulting from misaligned energy levels between the enzyme and the conducting material and their poor operational stability related to the overtime denaturation and activity drops of the enzyme have restricted any practical applications of these devices. ${ }^{14,15}$ In addition, weak bio-electronic coupling and limited mass transport restrain the performance of the EFCs. ${ }^{12,15,16}$ Evidently, there is a need for easily processable and stable electronic materials that embody seamless electronic communication with enzymes for high and long-lasting power.

To address these issues, we present a hydrophilic n-type (electron transporting) organic semiconductor for detecting glucose that can generate, from bodily fluids, the energy required to run basic circuit components. These devices leverage the high volumetric capacitance of the polymer film, its ability to accept and transport electrons as well as the electronic coupling of the conjugated unit with the enzyme facilitated by the ethylene glycol side-chains exposed at the outermost surface of the film. The sensor is a miniaturized organic electrochemical transistor (OECT) that comprises the n-type polymer at the gate electrode and in the channel. The power 
generator is an all polymer-based biofuel cell assembled on flexible substrates. We employ the GOx adsorbed polymer film as the anode in an EFC configuration, in conjunction with a polymeric, enzyme- and mediator-free cathode which undergoes $\mathrm{O}_{2}$ reduction reaction. Implementing a sensitive and robust OECT sensor that can be indefinitely powered by glucose is cost-effective and efficient and presents a portable solution for building self-reliant devices that serve the needs of next-generation biomedical devices.

The electron transporting material that we use is an NDI-T2 copolymer (named hereafter as P$90)^{17}$, consisting of an alternating naphthalene dicarboximide (NDI) acceptor and bithiophene (T2) donor subunits with randomly distributed alkyl and ethylene glycol side chains (Fig. 1A). The ratio of ethylene glycol to non-polar branched alkyl side chains in the composition is optimized (90:10) to enable adequate swelling of the polymer in water and therefore the electrochemical activity in aqueous electrolytes. Our OECT consists of P-90 patterned at the micron-scale channel and the lateral gate electrode that are both covered with a drop of the electrolyte of interest (Fig. 1A). This P-90 based, electrolyte-gated transistor operates in the accumulation mode, where a positive voltage at the gate electrode increases the channel current, switching the transistor ON (Fig. S1). The distinguishing property of an OECT is the volumetric interaction of electrolyte ions with the bulk of the film in the channel, leading to a substantial change in its doping state and electrical conductivity. ${ }^{18}$ As a result of this volumetric transduction, OECTs show record-high transconductance compared to other transistor technologies and thus are powerful biosensors. ${ }^{18}$ The high gain of OECTs translates into local amplification, allowing to build miniaturized sensors. ${ }^{19}$ P-90 film has an electronic mobility of $\sim 2 \times 10^{-4} \mathrm{~cm}^{2} \mathrm{~V}^{-1} \mathrm{~s}^{-1}$ and a high volumetric capacitance of $\sim 200{\mathrm{~F} . \mathrm{cm}^{-3}}^{-3}$, which yields high transconductance. ${ }^{20}$ To render the P-90 OECT selective to glucose, we drop cast the enzyme on the active area (on top of the P-90 channel and 
gate) that neither incorporated an exogenous electron mediator nor was chemically treated to immobilize the enzyme.

\section{N-type OECTs for glucose detection}

Fig. 1B shows the real-time response of the P-90 OECT coupled with GOx to glucose. In this experiment, we monitor the drain current, $I_{\mathrm{D}}$, measured at a constant source-drain voltage $\left(V_{\mathrm{D}}\right)$ and gate voltage $\left(V_{\mathrm{G}}\right)$ as successive amounts of glucose are added to the solution. $I_{\mathrm{D}}$ increases in a steplike manner following the increase in glucose concentration in the buffer. In accumulation mode transistors, it is the increase in $V_{\mathrm{G}}$, which causes an increase in the conductance of the channel. This is because a higher $V_{\mathrm{G}}$ pushes more cations into the channel to compensate for the electrons injected from the contacts. In our experiment, however, the $V_{\mathrm{G}}$ (as well as $V_{\mathrm{D}}$ ) is constant and the only variable is the glucose added to the electrolyte. Clearly, glucose causes an increase in the number of electrons injected to the P-90 channel functionalized with GOx. As the concentration of the analyte catalyzed by the enzyme increases, more cations are injected from the electrolyte into the channel to compensate for the larger quantity of the electronic charge. The dynamic range of this device extends from $10 \mathrm{nM}$ to $20 \mathrm{mM}$ of glucose. The low detection limit of $10 \mathrm{nM}$ is comparable to other OECTs employing functionalized gate electrodes, yet here it is achieved without any functionalization processes or external electron shuttles. ${ }^{21,22}$ When $20 \mathrm{mM}$ of glucose solution is replaced with the buffer solution on top of the device, the current reverts to its original value. Extended Data Fig. 1 shows that the biosensor exhibits excellent reversibility and minimal hysteresis when exposed to low concentrations of glucose $(10 \mathrm{nM}$ to $100 \mu \mathrm{M})$, while at higher concentrations ( $\mathrm{mM}$ range), the response to decremental glucose concentration is no longer reversible. To investigate the effect of enzymatic reaction on the electrical properties of the P-90 film, we disconnect the gate electrode and measure the changes in the current flowing in the 
channel after functionalization with GOx and addition of glucose in the buffer. Fig. 1C shows that with $1 \mathrm{mM}$ of glucose in the solution, the current output of the chemiresistor is $\sim 3 \mathrm{nA}$. In the OECT configuration, the output of the very same film is amplified to more than 45 nA (Fig. 1B), highlighting the advantage of the OECT circuitry.

Figure 1. Glucose sensing with an n-type accumulation mode OECT

a
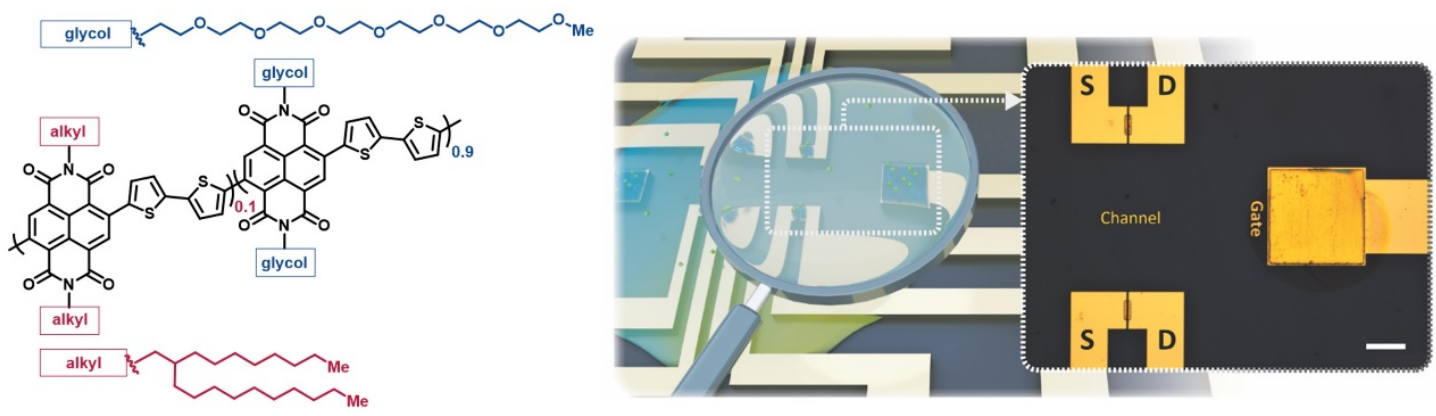

b

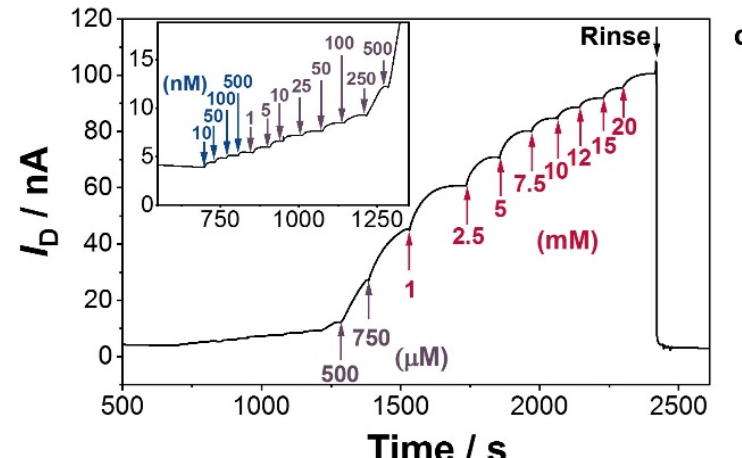

d

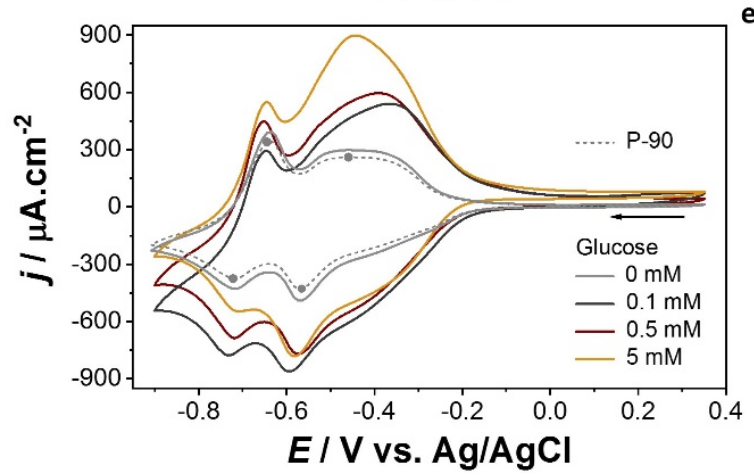

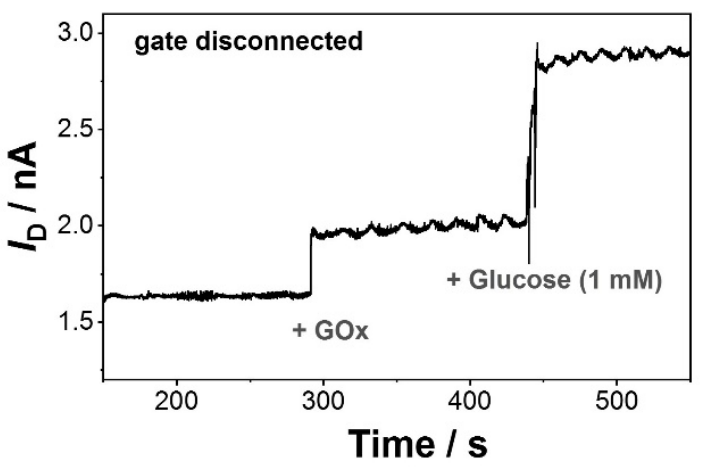

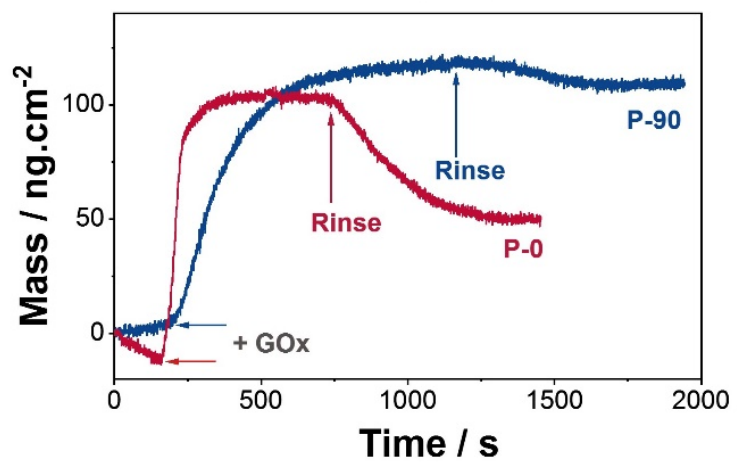


To compare the glucose-affinity of our sensor to those reported in the literature, we calculated the Michaelis-Menten constant $\left(K_{\mathrm{m}}^{\text {app }}\right) \cdot{ }^{23}$ We find that $K_{\mathrm{m}}$ app equals $1.73 \mathrm{mM}$, a value significantly smaller than other electrochemical glucose sensors (Table S1), evidencing the strong binding affinity of the enzyme adsorbed on P-90 to the glucose in the electrolyte. We further probe the electrocatalysis in the P-90/GOx system using cyclic voltammetry (CV). After casting GOx on P90, we observe an enhancement in both the anodic and cathodic currents with no apparent shifts in the peak potentials of the redox couples characteristic to the NDI backbone (Fig. 1D). ${ }^{24}$ Following the addition of glucose in the solution, the CV curve of the film undergoes significant changes involving an increase in the amplitudes of all redox peaks. The enzymatic reaction has the same effect on the CV curve of P-90/GOx film when recorded in air-equilibrated solutions as well as in $\mathrm{O}_{2}$-free solutions (Fig. S2), evidencing that the oxidation of glucose in this system is not $\mathrm{O}_{2}$ mediated. ${ }^{25}$ Moreover, the P-90 OECT displays limited sensitivity to $\mathrm{H}_{2} \mathrm{O}_{2}$ (Extended Data Fig. 2). When we use a denatured GOx to functionalize the film, the resulting OECTs are no longer sensitive to glucose (Fig. S3). Considering the limited porosity of the P-90 film (Fig. S4), the enzyme is not expected to penetrate the polymer but is, instead, located on top of the film. Indeed, a substantial amount of GOx remains on top of P-90 after being rinsed with PBS (Fig. 1E). On the contrary, most of the enzyme initially adsorbed on the ethylene glycol free analogue of P-90, that is P-0 (the ratio of glycol: alkyl side chains is 0:100), is washed away. The presence of ethylene glycol content of the NDI-T2 film is thus critical for establishing interactions between the protein and the polymer, ${ }^{26}$ enabling the functionalization of P-90 with GOx.

These results suggest that the increase in the drain current of the OECT is due to the direct electrical communication of GOx with the polymer film and its reaction with glucose, a spontaneous biological event serving as the manually exerted gate voltage. The P-90 film accepts electrons of 
the enzymatic reaction and transports them along its backbone (see the possible reactions in the P90/GOx system summarized in Fig. S5). Further evidence for this mechanism comes from the optical absorption spectrum of P-90 which displays distinct features associated with its doping state. When P-90 undergoes from a neutral to an electrochemically reduced state (doped by cations in PBS), the intensity of the low energy absorption feature decreases while a new peak around 450 nm arises (Fig. 2A). ${ }^{17,27}$ Fig. 2B and Extended Data Fig. 3A show that similar changes occur for the P-90/GOx system - yet these are triggered by the addition of glucose into the solution. $\mathrm{H}_{2} \mathrm{O}_{2}$, on the other hand, has no such effect on the spectrum of P-90 (Extended Fig. 3B), just as the OECT having no sensitivity to $\mathrm{H}_{2} \mathrm{O}_{2}$ (Extended Data Fig. 2). The enzymatic reaction perturbs the electronic structure of the polymer, emulating the doping voltage. To track these events at the molecular level, we recorded the Raman spectra of our system during electrochemical doping and the course of enzymatic reactions. Extended Data Fig. 3C and Fig. 2C display the evolution of the Raman spectrum of a P-90 film subject to increasing doping potentials. 
Figure 2. As the enzyme catalyzes glucose, the n-type polymer gets doped as if it is electrochemically addressed.

a
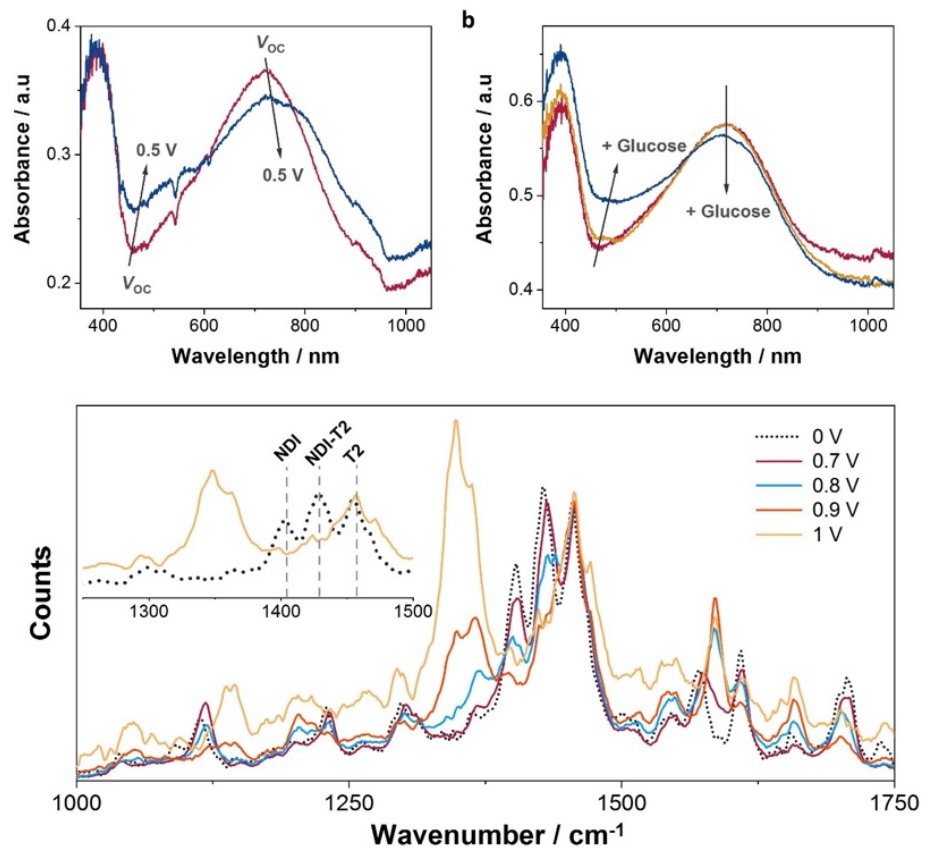

d

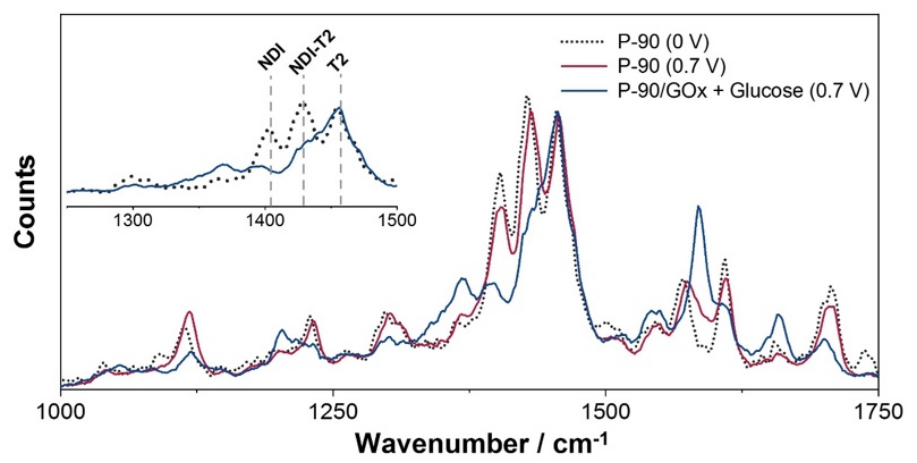

When the film is doped with electrolyte ions, its spectral profile shows peaks that have changed in intensity and position and the extent of these changes increases with the doping voltage, suggesting that structural rearrangements occur concurrently with the localization of the $\pi$-electrons on the backbone (Fig. 2C, see discussion in Supplementary Information). The switch in the electrochemical state affects mainly the Я-mode of the NDI unit: ${ }^{28,29}$ the characteristic peak at 
$1407 \mathrm{~cm}^{-1}$ reduces in intensity as the doping voltage increases up to $1 \mathrm{~V}$. Meanwhile, two lower frequency peaks located at 1347 and $1364 \mathrm{~cm}^{-1}$ gain in intensity and dominate the spectrum. These peaks are to be attributed to the dopant cations that generate strongly localized defects, transforming the $\mathrm{T} 2$ structure from an aromatic to a quinoid form. As the voltage is reverted to 0 V, the spectrum recovers its original shape (Extended Data Fig. 3C). Upon adsorption of the enzyme on the P-90 film, we observe a minor increase in the intensity of the $\mathrm{C}=\mathrm{O}, \mathrm{C}=\mathrm{C}$, and $\mathrm{C}=\mathrm{N}$ peaks of NDI unit while the Я-modes remain unaffected (Extended Data Fig. 3D). As glucose is introduced to the solution, we see changes similar to those triggered by electrochemical doping (although the voltage is kept constant at $0.7 \mathrm{~V}$ ): Я-mode on the NDI loses its intensity while the neighboring lower energy peaks become prominent (Fig. 2D). From these results, we conclude that the active sites of the enzyme and the copolymer have an interface which leads to an efficient electronic communication. At this bio-electronic interface, analyte oxidation increases the conductivity of the polymer by donating new electrons to the backbone, and this process proceeds without the aid of an external electron mediator.

One distinguishing property of an n-type transistor is that the device is operated at positive gate and drain voltages. Since both polymer gate and channel are functionalized with GOx, these operating conditions enable us to control the location of the enzymatic reaction by modulating the polarity of interfaces with respect to each other. Fig. 3A depicts the calibration curves at different operation conditions where we varied the magnitude of the bias applied at the channel $\left(V_{\mathrm{D}}\right)$ in relation to a constant gate potential $\left(V_{\mathrm{G}}\right)$. In all conditions, the devices exhibit the same dynamic range and the channel current scales linearly with glucose concentration up to $100 \mu \mathrm{M}$ with a second linear region appearing for higher glucose concentrations. What differs is the sensitivity of a specific analyte concentration range. The sensitivity towards low glucose concentrations is 
highest when the device operates at $V_{\mathrm{D}}>V_{\mathrm{G}}\left(\right.$ Fig. 3A). When $V_{\mathrm{D}}>V_{\mathrm{G}}$, we expect that the enzymatic reaction transfers electrons to the channel because the area close to the drain contact is more positively biased than the gate (Fig. 3B, see discussion in Fig. S6). On the other hand, when $V_{\mathrm{G}}$ is higher or equal to $V_{\mathrm{D}}$, the device exhibits greater sensitivity to high glucose levels ( $\mu \mathrm{M}-\mathrm{mM}$ range). Moreover, without GOx functionalization, the P-90 OECT is not sensitive to glucose (Fig. 3C). When functionalized with GOx, the device exhibits excellent selectivity to glucose and is markedly less sensitive to most common interferences at their physiologically relevant concentrations. The same OECT measures glucose content in saliva samples collected from healthy patients, yielding a linear response to glucose at concentrations relevant for non-diabetic and diabetic saliva (from $28 \mu \mathrm{M}$ to $0.85 \mathrm{mM}$ ) (Fig. 3D).

Figure 3. Biosensor performance at different operating conditions and in the presence of endogenous species

a

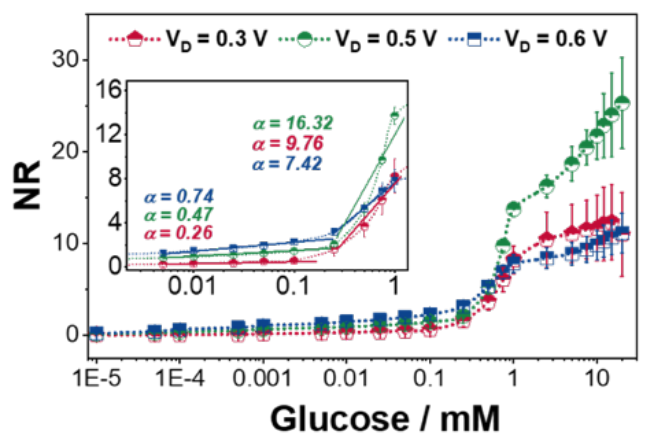

c

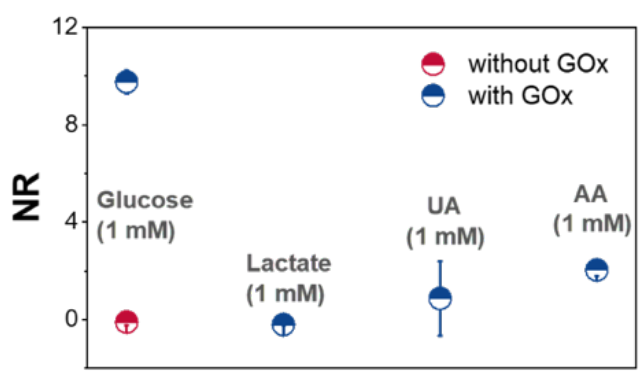

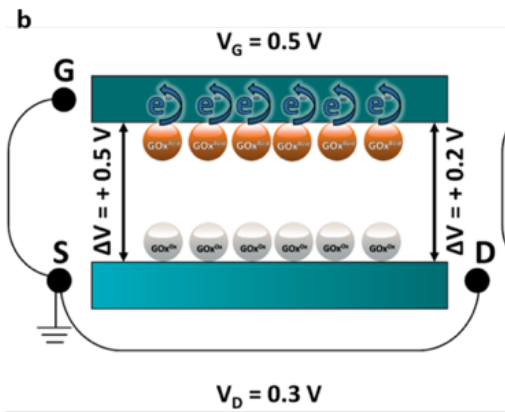

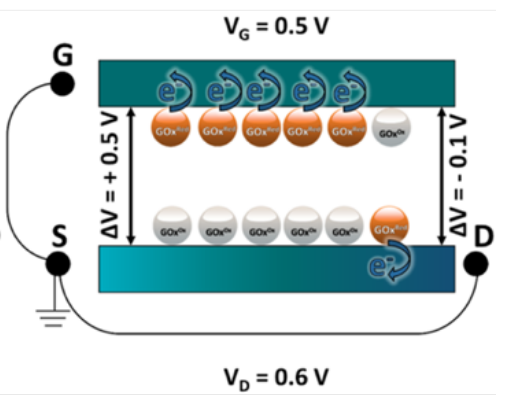

d

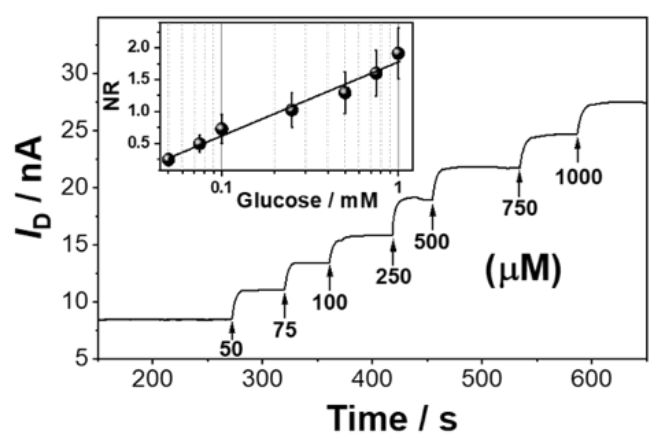




\section{N-type biofuel cells for power generation from glucose}

Since P-90/GOx film generates current upon the catalytic reaction of GOx with glucose, it can be employed as an anode for an enzymatic biofuel cell (EFC) (Fig. 4A). The reaction produces electrons that are transferred to the P-90 anode (Fig. 4B). These electrons then travel through the external circuit to the cathode which reduces dioxygen to water so that the circuit generates power from glucose and $\mathrm{O}_{2} .{ }^{12}$ Our cathode is $\mathrm{p}$ (EDOT-co-EDOTOH), a p-type copolymer that we electropolymerized on a gold-coated surface with an area identical to the anode (Fig. S7). We selected this material as the cathode due to the well-known ability of PEDOT derivatives to reduce $\mathrm{O}_{2},{ }^{30-33}$ as well as the simplicity and low cost of fabrication. Our P(EDOT-co-EDOTOH) exhibits $\mathrm{O}_{2}$ reduction reaction (ORR) as evidenced by the enhancement of the reduction current in $\mathrm{O}_{2}$ saturated environments (Extended Data Fig. 4). The potential difference that corresponds to the onset potentials of the glucose oxidation and $\mathrm{O}_{2}$ reduction potentials of our EFC is evaluated by $\mathrm{CV}$ experiments $\left(\mathrm{E}_{\text {cell }}=\sim 0.3 \mathrm{~V}\right)$ and shown in Extended Data Fig. 5. The polymeric cathode leads to a higher open circuit voltage (OCV) compared to Pt, a common cathode of biofuel cells (Fig. S8), and exhibits good stability against continuous cycling with a capacitance retention of $93 \%$ upon $100 \mathrm{CV}$ cycles (Fig. S9A). A scan rate dependence study of the current of the bioanode generated in the presence of glucose reveals a linear relationship between the current and the scan rate, indicative of surface-controlled processes (Fig. S10). ${ }^{34,35}$ Elucidating this curve, we extracted the heterogeneous electron transfer rate constant, $k_{\mathrm{ET}}$, following the Laviron model (Table S2). ${ }^{34,36}$ The high $k_{\mathrm{ET}}\left(8.11 \mathrm{~s}^{-1}\right)$ advocates on the effectiveness of the polymer in accepting and transporting electrons generated during the glucose oxidation. Notably, the P-90 film remains stable upon consecutive cycling (100 cycles) with a current retention greater than 97\% (Fig. S9B). 
Figure 4. The performance of the all-polymer biofuel cell

a

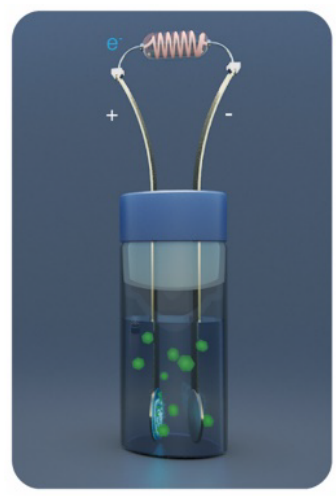

c

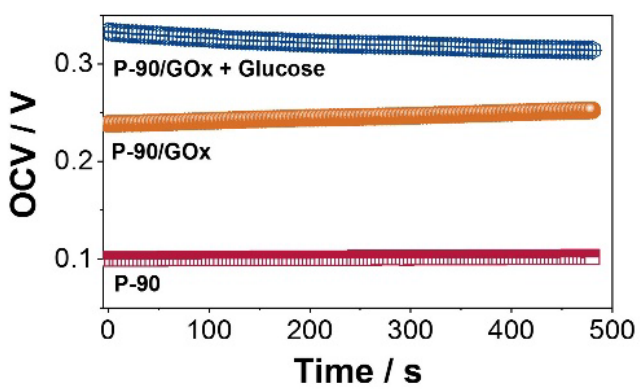

b

Cathode (-)
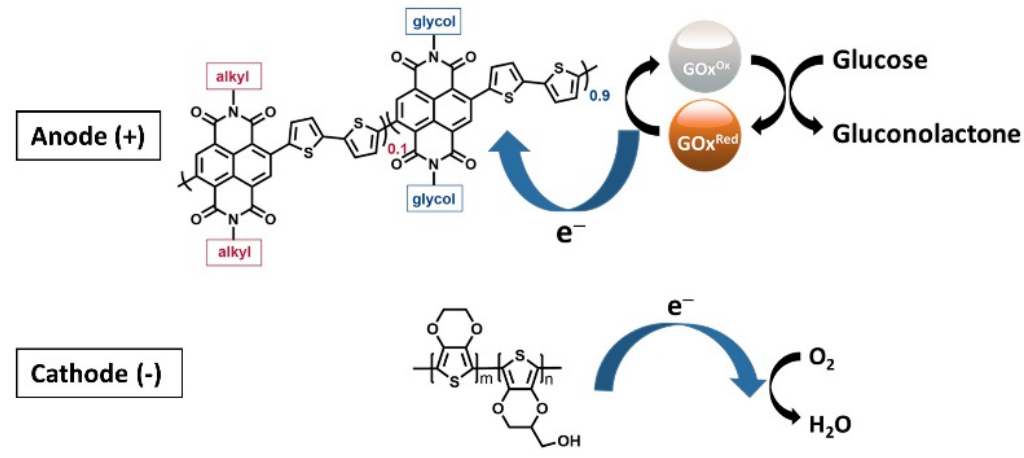

d

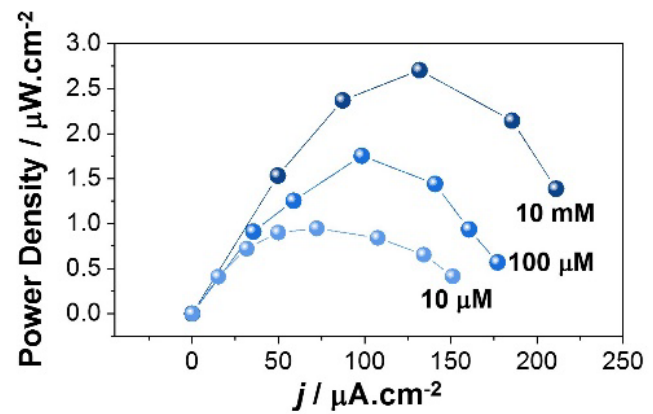

Fig. 4C portrays the evolution of open circuit voltage in the absence and co-presence of the enzyme and glucose. When electrically connected in aqueous media, the two polymer films act as efficient catalysts and harvest glucose and $\mathrm{O}_{2}$ to generate power. The power density curves of the $\mathrm{EFC}$ are depicted in Fig. 4D and Extended Data Fig. 6. As glucose is introduced in the solution, we observe a clear increase in the power generated by the EFC, reaching a maximum of $2.8 \mu \mathrm{W} \cdot \mathrm{cm}^{-2}$ for $10 \mathrm{mM}$ of glucose. Given the simple assembly of our electrodes, we explored the possibility of both incorporating and omitting a membrane (Nafion) to separate the anodic and cathodic compartments. The membrane-free cell had a lower maximum power density (MPD, $0.4 \mu \mathrm{W} . \mathrm{cm}^{-}$ ${ }^{2}$ for $10 \mathrm{mM}$ of glucose) than the membrane-based EFC (Extended Data Fig. 7) despite its reduced internal resistance (Fig. S11). Taken together, the polymers selected as the anodic and cathodic 
coatings exhibit intrinsic catalytic properties on a level competitive with the other reported systems - which have undergone exhaustive device performance optimization - while benefiting from the ease of electrode preparation (Table S3). Nonetheless, one poignant characteristic of n-type semiconducting polymer-based devices is their low electrical conductivity, which contributes here significantly to the device internal resistance and hence hindering a high-power output. Indeed, the MPD increases $\sim 60$-fold for the EFC comprising an electrochemically doped P-90 at the anode (Extended Data Fig. 8). Using the semiconducting polymer at the anode in its conducting form is a simple demonstration of how the performance of this all-polymer EFC can be improved. Other optimization methods include doping the n-type film with molecular dopants, ${ }^{37}$ increasing the planarity of its backbone, ${ }^{38}$ turning to polymer composites with conducting particles, ${ }^{39}$ controlling the ordering and multi-scale assembly of the chains via processing means, ${ }^{40}$ to name a few.

We next investigated the stability of the EFCs by monitoring the OCV. We also examined the behavior under non-equilibrium conditions (beyond the OCV) when the cell is biased at high positive voltages $(>0.6 \mathrm{~V})$. At this extended biasing regime, we find that both membrane-free and membrane-based EFCs produce a high amount of $\mathrm{H}_{2} \mathrm{O}_{2}$ responsible for the large currents generated (Fig. S12). ${ }^{41}$ After 30 days of use, the OCV drops to $30 \%$ of its original value while the EFC preserves $\sim 40 \%$ of the power that it produces when biased up to the $\mathrm{H}_{2} \mathrm{O}_{2}$ production regime (Fig. S13A). If the enzyme is replenished between measurements, the device can be used for additional 20 days (Fig. S13B). Our stability studies revealed that in the current configuration, the EFC stability is mainly challenged by enzyme denaturation and instability of the p-type polymer coating. Fig. S13C shows that the stability can be improved when the P-90/GOx film is encapsulated by Nafion coating (EFC maintains $45 \%$ of its OCV after 30 days of use). As for the 
membrane-free devices, their stability was on par with the ones having a membrane (Fig. S14), attesting their ability to generate the power to operate a sensor or actuator continuously.

Figure 5. Performance of the EFC as a self-powered sensor and as a circuit component.

a

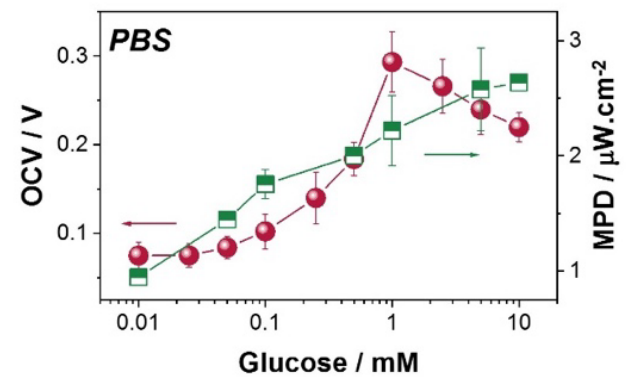

C

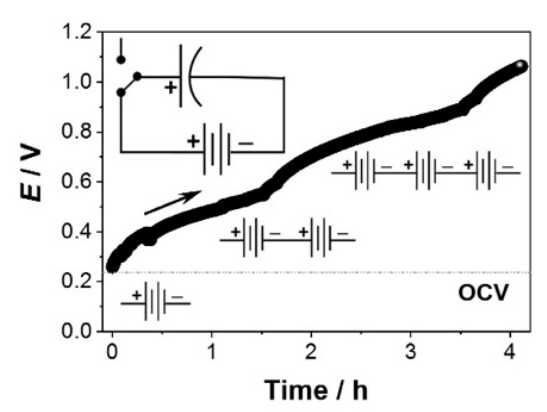

b

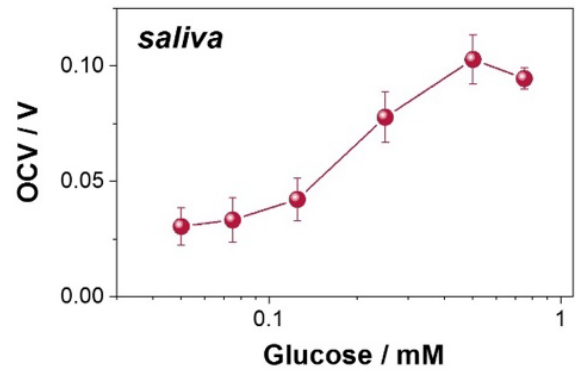

d

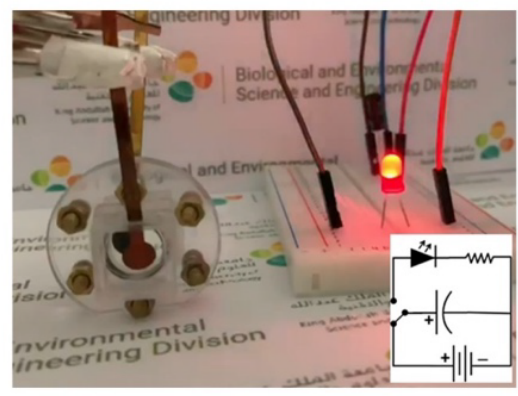

e
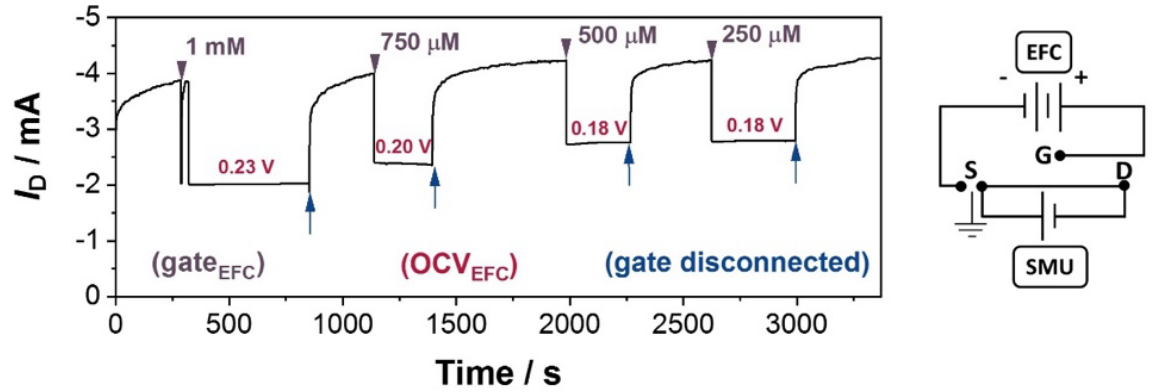

We next monitored the change in the OCV and MPD of our biofuel cell as a function of glucose concentration in saliva and PBS (Fig. 5A and 5B). Both the OCV and the MPD increase with 
glucose concentration, demonstrating the use of our EFC as a self-powered glucose sensor in physiological fluids. Membrane-free devices follow a similar trend, validating the glucose activated power generation of our EFCs (Extended Fig. 7B). To this end, we connected three EFCs in series polarizing a capacitor $(100 \mu \mathrm{F})$ to draw a total output of $1.1 \mathrm{~V}$ in PBS (Fig. 5C). When fueled by $1 \mathrm{mM}$ glucose in PBS, this EFC platform was able to drive an LED, as shown in Fig. 5D and video S1. The membrane-free EFC configuration also effectively powered the device as the bioanode and cathode were immersed in PBS containing glucose (Fig. S15, video S2). What's more, we used a membrane-free EFC to switch ON and OFF an OECT in a fully-integrated platform (Fig. 5E). The channel of this OECT is made of PEDOT:PSS and has the same geometry as our OECT biosensor. In contrast, it operates in depletion mode, meaning that the drain current decreases upon application of a gate voltage. As the EFC provides the power to apply a voltage at the gate electrode, the drain current decreases, consistent with the OECT operation. Upon supplying more glucose to the EFC, the OECT current decreases further. As such, we capitalize on the dependence of the $\mathrm{OCV}$ on glucose concentration to control the gate voltage. The power of EFCs can also be used to bias both the gate and the channel simultaneously (Extended Data Fig. $9)^{42}$.

\section{Outlook}

We demonstrated the use of an n-type polymer in a miniaturized OECT for detection of glucose and in an EFC for generation of power from physiological fluids. The sensor presents a wide dynamic range from $10 \mathrm{nM}$ to $20 \mathrm{mM}$ of glucose with a sensitivity tunable by varying the biasing conditions. The presence of ethylene glycol on the side-chains of the polymer enables the adsorption of the enzyme onto the polymer surface, allowing a seamless bio-electronic coupling. 
The enzymatic reaction serves as a biological switch which acts on the channel conductance, and this process spontaneously proceeds in the absence of exogenous electron mediator.

Going one step forward, using the n-type polymer as the anode, we assembled an enzymatic biofuel cell that consumes green fuel such as glucose and $\mathrm{O}_{2}$. The device is made of polymeric electrodes (mediator-free) assembled on flexible substrates and can function with or without a membrane. Drawing its energy from glucose present naturally in all bodily fluids, this EFC provides sufficient voltage to drive OECTs and other circuit elements. The EFC output scales with the glucose content of saliva, demonstrating its ability to perform as a self-powered glucose sensor in complex media. While displaying over a month-long stability in terms of power output and $\mathrm{OCV}$, in its most uncomplicated design, this all-polymer, biofuel-fed power source competes with numerous previously reported systems, offering a solution for autonomous biosensing while benefiting from the ease of preparation. This configuration validates our strategy to fabricate lowcost, stable, polymeric biofuel cells that utilize glucose to power other electronic devices. 


\section{Figure Captions}

Figure 1. Glucose sensing with an n-type accumulation mode OECT. (A) The chemical structure of the n-type copolymer P-90. Schematic illustration of the sensor (gate dimensions: $500 \times 500 \mu \mathrm{m}^{2}$, channel dimensions: $100 \mu \mathrm{m}$ (width) $\times 10 \mu \mathrm{m}$ (length)). The OECT active area was incubated with GOx solution before measurements. The active area in the illustration contains 6 channels and 2 gate electrodes made of P-90. The microscope image shows two of the P-90 channels alongside a P-90 gate electrode. Scale bar is $200 \mu \mathrm{m}$. (B) Real-time response of the OECT (source-drain current, $I_{\mathrm{D}}$, as a function of time) as successive amounts of glucose were added to the buffer. The gate and drain potentials were $0.5 \mathrm{~V}$. Inset shows the real-time response of the sensor to low concentrations of glucose $(\leq 500 \mu \mathrm{M})$. (C) The effect of GOx and glucose oxidation by GOx on P-90 current. The gate was disconnected, and a source-drain bias (0.3 V) was applied to the polymer film in the channel. (D) Cyclic voltammogram of a P-90 electrode in the absence and presence of GOx. The marked points in the P-90 curve denote two quasi-reversible redox peaks located at $-0.57 \mathrm{~V} /-0.44 \mathrm{~V}$ and $-0.73 \mathrm{~V} /-0.65 \mathrm{~V}$ (reduction/oxidation), characteristic of the NDI backbone. As glucose concentration increases, the oxidation current increases further accompanied by a decrease in the reduction current. The scan rate is $50 \mathrm{mV} \cdot \mathrm{s}^{-1}$ and the solution is PBS. The arrow indicates the scan direction. (E) QCM-D measurements tracking the interactions between GOx and the two polymer films differing with their glycol content (P-90; ratio of glycol: alkyl side chains is 90:10, and P-0; ratio of glycol: alkyl side chains is 0:100) during two stages: (1) when the enzyme was injected into the PBS solution (+ GOx) and (2) when the P-90/GOx film was rinsed with PBS (Rinse). 
Figure 2. As the enzyme catalyzes glucose, the n-type polymer gets doped as if it is electrochemically addressed. (A) UV-VIS spectrum of a P-90 film in the absence and presence of a doping bias (0.5 V vs. Ag/AgCl) in PBS. (B) UV-VIS spectrum of a P-90 film (red), P-90/GOx film (yellow) and P-90/GOx film in the presence of $1 \mathrm{mM}$ of glucose (blue). All films are subject to a doping potential at $0.5 \mathrm{~V}$ vs $\mathrm{Ag} / \mathrm{AgCl}$. (C) Raman spectrum of a P-90 film at $0 \mathrm{~V}$ (de-doped) as well as when it is biased at increasing doping potentials (0.7 - 1 V). See Extended Data Figure S3C for a full range of doping potentials and the main peak attributions of the P-90 film. Inset shows the Raman spectra zoomed in the $1250-1500 \mathrm{~cm}^{-1}$ region and the peak attributions therein. (D) Raman spectrum of a P-90 film (0 V), reduced P-90 film (0.7 V) and a P-90/GOx film in the presence of $1 \mathrm{mM}$ of glucose $(0.7 \mathrm{~V})$. Inset shows the Raman spectra zoomed in the 1250-1500 $\mathrm{cm}^{-1}$ region where the enzymatic reaction leads to predominant alterations. All Raman data were normalized to the Я-mode peak intensity of the bithiophene unit at $1457 \mathrm{~cm}^{-1}$ (see Methods for more details).

Figure 3. Biosensor performance at different operating conditions and in the presence of endogenous species. (A) Normalized response (NR) of the OECT biosensor to glucose. The devices were operated at various drain voltages $\left(V_{\mathrm{D}}\right)$ and a constant gate voltage $\left(V_{\mathrm{G}}\right)$ of $0.5 \mathrm{~V}$ so that $V_{\mathrm{G}}>V_{\mathrm{D}}(\mathrm{red}), V_{\mathrm{D}}>V_{\mathrm{G}}$ (blue) and $V_{\mathrm{D}}=V_{\mathrm{G}}$ (green). $\alpha$ is the slope of the linear fits at two distinct concentration ranges. The log scale was introduced to improve visualization of the response to low concentrations. Inset: zoom-in of the calibration curves at low glucose concentration regime. The sensitivity can be tuned for detection at a particular concentration range simply by changing the operating conditions, alleviating the need to change the device design. (B) Schematic for the location of the enzymatic reaction based on the operating conditions. The electronic wiring takes place only at the gate $\left(V_{\mathrm{G}}>V_{\mathrm{D}}\right)$ and also at the channel $\left(V_{\mathrm{D}}>V_{\mathrm{G}}\right)$. (C) NR 
of the OECT biosensor to the most common interferences in biological fluids. The species were introduced to the measurement solution at their physiologically relevant concentrations. The sensor does not respond to glucose when the active area is not functionalized with GOx (red symbol). A somewhat marginal current response is detected for uric and ascorbic acids. This interference current can be accounted for by using a blank channel/gate via common circuit engineering approaches. (D) Real-time response of the OECT to successive amounts of glucose added into the saliva as the measurement solution. Inset depicts the calibration curve. Experiments were performed three times for each glucose concentration. In all experiments, error bars represent the standard deviation from three different devices.

Figure 4. The performance of the all-polymer biofuel cell. (A) Schematic of a membrane-free EFC where flexible Au-coated polyimide is used as substrate that carries cathode or anode materials. The EFC comprises P-90/GOx at the anode and p(EDOT-co-EDOTOH) at the cathode. Note that no mediators are integrated in the cathode or the anode while the cathode is not relying on an enzyme. The electrolyte is the PBS ( $\mathrm{pH}$ 7.2) or saliva solution containing glucose (B) Reactions occurring during the operation of the EFC. (C) OCV of the EFC comprising P-90 alone and when it is functionalized with GOx as well as when the cell is fueled by $1 \mathrm{mM}$ of glucose in PBS. (D) Power output of the EFC when the anode is functionalized with GOx (inset) as well as in the presence of selected concentrations of glucose as a function of current density. The data were obtained by varying the circuit load. The anodic and cathodic compartments of the EFC are separated by a Nafion membrane.

Figure 5. Performance of the EFC as a self-powered sensor and as a circuit component. Performance of the EFC (with Nafion membrane separator) operated with a broad range of glucose 
concentrations in (A) PBS and (B) saliva. Since glucose is oxidized at the anode by GOx, the current generated by this reaction is proportional to analyte concentration along with the power output of the biofuel cell. At higher glucose concentrations, OCV decreases because of mass transport limitations. Error bars represent the standard deviation from three different devices. (C) The output voltage of the EFC charging a capacitor $(100 \mu \mathrm{F})$ as a function of time. With prolonged charging, an array of three EFCs in series was introduced. Inset shows the circuit connection of the polarized capacitor. (D) Digital photograph of the EFC experimental setup switching ON an LED; inset illustrates the configuration of this electrical circuit. (E) Real-time response of a PEDOT:PSS OECT gated with a membrane-free EFC that is fueled by a range of glucose concentrations. The gate electrode was disconnected from the electrolyte while changing the glucose concentration in the EFC cell. The right panel represents the equivalent electronic circuit. Note that we decrease the glucose content to show that the device performance is not dependent on a stepwise increase in biofuel concentration. 


\section{References}

1 Pappa, A.-M. et al. Organic Electronics for Point-of-Care Metabolite Monitoring. Trends Biotechnol 36, 45-59, doi:https://doi.org/10.1016/i.tibtech.2017.10.022 (2018).

2 Gifford, R. Continuous Glucose Monitoring: 40 Years, What We've Learned and What's Next. ChemPhysChem 14, 2032-2044, doi:doi:10.1002/cphc.201300172 (2013).

3 Witkowska Nery, E., Kundys, M., Jeleń, P. S. \& Jönsson-Niedziółka, M. Electrochemical Glucose Sensing: Is There Still Room for Improvement? Anal. Chem 88, 11271-11282, doi:10.1021/acs.analchem.6b03151 (2016).

4 Wang, J. Electrochemical Glucose Biosensors. Chem. Rev. 108, 814-825, doi:10.1021/cr068123a (2008).

$5 \quad$ Heller, A. \& Feldman, B. Electrochemical Glucose Sensors and Their Applications in Diabetes Management. Chem. Rev. 108, 2482-2505, doi:10.1021/cr068069y (2008).

$6 \mathrm{Wu}, \mathrm{Y} . \& \mathrm{Hu}, \mathrm{S}$. Biosensors based on direct electron transfer in redox proteins. Microchim. Acta 159, 1-17, doi:10.1007/s00604-007-0749-4 (2007).

7 Zhang, W. \& Li, G. Third-Generation Biosensors Based on the Direct Electron Transfer of Proteins. Anal. Sci. 20, 603-609, doi:10.2116/analsci.20.603 (2004).

8 Zhu, C., Yang, G., Li, H., Du, D. \& Lin, Y. Electrochemical Sensors and Biosensors Based on Nanomaterials and Nanostructures. Anal. Chem 87, 230-249, doi:10.1021/ac5039863 (2015).

9 Kissinger, P. T. Engineering the Bioelectronic Interface: Applications to Analyte Biosensing and Protein Detection. J. Am. Chem. Soc. 132, 1444-1444, doi:10.1021/ja9108648 (2010).

10 Banica, F. G. Chemical Sensors and Biosensors: Fundamentals and Applications. (Wiley, 2012).

11 Simon, D. T., Gabrielsson, E. O., Tybrandt, K. \& Berggren, M. Organic Bioelectronics: Bridging the Signaling Gap between Biology and Technology. Chem. Rev. 116, 13009-13041, doi:10.1021/acs.chemrev.6b00146 (2016).

12 Luz, R. A. S., Pereira, A. R., de Souza, J. C. P., Sales, F. C. P. F. \& Crespilho, F. N. Enzyme Biofuel Cells: Thermodynamics, Kinetics and Challenges in Applicability. ChemElectroChem 1, 1751-1777, doi:doi:10.1002/celc.201402141 (2014).

13 Minteer, S. D., Atanassov, P., Luckarift, H. R. \& Johnson, G. R. New materials for biological fuel cells. Mater. Today 15, 166-173, doi:https://doi.org/10.1016/S1369-7021(12)70070-6 (2012).

14 Aghahosseini, H. et al. Glucose-based Biofuel Cells: Nanotechnology as a Vital Science in Biofuel Cells Performance. Nanochem Res 1, 183-204, doi:10.7508/ncr.2016.02.006 (2016).

15 Kwon, C. H. et al. High-power hybrid biofuel cells using layer-by-layer assembled glucose oxidasecoated metallic cotton fibers. Nat. Commun. 9, 4479, doi:10.1038/s41467-018-06994-5 (2018).

16 Wen, D. \& Eychmüller, A. Enzymatic Biofuel Cells on Porous Nanostructures. Small 12, 4649-4661, doi:doi:10.1002/smll.201600906 (2016).

17 Giovannitti, A. et al. The Role of the Side Chain on the Performance of N-type Conjugated Polymers in Aqueous Electrolytes. Chem. Mater. 30, 2945-2953, doi:10.1021/acs.chemmater.8b00321 (2018).

18 Inal, S., Rivnay, J., Suiu, A.-O., Malliaras, G. G. \& McCulloch, I. Conjugated Polymers in Bioelectronics. Acc. Chem. Res. 51, 1368-1376, doi:10.1021/acs.accounts.7b00624 (2018).

19 Spyropoulos, G. D., Gelinas, J. N. \& Khodagholy, D. Internal ion-gated organic electrochemical transistor: A building block for integrated bioelectronics. Sci. Adv. 5, eaau7378, doi:10.1126/sciadv.aau7378 (2019).

20 Pappa, A. M. et al. Direct metabolite detection with an n-type accumulation mode organic electrochemical transistor. Sci. Adv. 4, doi:10.1126/sciadv.aat0911 (2018). 
21 Liao, C., Zhang, M., Niu, L., Zheng, Z. \& Yan, F. Highly selective and sensitive glucose sensors based on organic electrochemical transistors with graphene-modified gate electrodes. J. Mater. Chem. B 1, 3820-3829, doi:10.1039/C3TB20451K (2013).

22 Piro, B. et al. Fabrication and Use of Organic Electrochemical Transistors for Sensing of Metabolites in Aqueous Media. Appl. Sci. 8, 928 (2018).

23 Lineweaver, H. \& Burk, D. The Determination of Enzyme Dissociation Constants. J. Am. Chem. Soc. 56, 658-666, doi:10.1021/ja01318a036 (1934).

24 Trefz, D. et al. Electrochemical Investigations of the N-Type Semiconducting Polymer P(NDI2ODT2) and Its Monomer: New Insights in the Reduction Behavior. J. Phys. Chem. C119, 22760-22771, doi:10.1021/acs.jpcc.5b05756 (2015).

25 Wooten, M., Karra, S., Zhang, M. \& Gorski, W. On the Direct Electron Transfer, Sensing, and Enzyme Activity in the Glucose Oxidase/Carbon Nanotubes System. Anal. Chem 86, 752-757, doi:10.1021/ac403250w (2014).

26 Zhang, Y. et al. Visualizing the Solid-Liquid Interface of Conjugated Copolymer Films Using Fluorescent Liposomes. ACS Appl. Bio Mater. 1, 1348-1354, doi:10.1021/acsabm.8b00323 (2018).

27 Giovannitti, A. et al. N-type organic electrochemical transistors with stability in water. Nat. Commun. 7, 13066, doi:10.1038/ncomms13066

https://www.nature.com/articles/ncomms13066\#supplementary-information (2016).

28 Giussani, E., Fazzi, D., Brambilla, L., Caironi, M. \& Castiglioni, C. Molecular Level Investigation of the Film Structure of a High Electron Mobility Copolymer via Vibrational Spectroscopy. Macromolecules 46, 2658-2670, doi:10.1021/ma302664s (2013).

29 Navarrete, J. T. L. \& Zerbi, G. Lattice dynamics and vibrational spectra of polythiophene. II. Effective coordinate theory, doping induced, and photoexcited spectra. J. Chem. Phys. 94, 965970, doi:10.1063/1.459987 (1991).

30 Mitraka, E. et al. Oxygen-induced doping on reduced PEDOT. J. Mater. Chem. A 5, 4404-4412, doi:10.1039/C6TA10521A (2017).

31 Singh, S. K., Crispin, X. \& Zozoulenko, I. V. Oxygen Reduction Reaction in Conducting Polymer PEDOT: Density Functional Theory Study. J. Phys. Chem. C 121, 12270-12277, doi:10.1021/acs.jpcc.7b03210 (2017).

32 Winther-Jensen, B., Winther-Jensen, O., Forsyth, M. \& MacFarlane, D. R. High Rates of Oxygen Reduction over a Vapor Phase-Polymerized PEDOT Electrode. Science 321, 671-674, doi:10.1126/science.1159267 (2008).

33 Mitraka, E. et al. Electrocatalytic Production of Hydrogen Peroxide with Poly(3,4ethylenedioxythiophene) Electrodes. Adv. Sustain. Syst. 3, 1800110, doi:10.1002/adsu.201800110 (2019).

34 Eckermann, A. L., Feld, D. J., Shaw, J. A. \& Meade, T. J. Electrochemistry of redox-active selfassembled monolayers. Coord. Chem. Rev. 254, 1769-1802, doi:https://doi.org/10.1016/j.ccr.2009.12.023 (2010).

35 Salimi, A., Sharifi, E., Noorbakhsh, A. \& Soltanian, S. Immobilization of glucose oxidase on electrodeposited nickel oxide nanoparticles: Direct electron transfer and electrocatalytic activity. Biosens. Bioelectron 22, 3146-3153, doi:https://doi.org/10.1016/j.bios.2007.02.002 (2007).

36 Laviron, E. General expression of the linear potential sweep voltammogram in the case of diffusionless electrochemical systems. J. electroanal. chem. interfacial electrochem. 101, 19-28, doi:https://doi.org/10.1016/S0022-0728(79)80075-3 (1979).

37 Liu, J. et al. Enhancing Molecular n-Type Doping of Donor-Acceptor Copolymers by Tailoring Side Chains. Adv. Mater. 30, 1704630, doi:doi:10.1002/adma.201704630 (2018). 
38 Wang, S. et al. A Chemically Doped Naphthalenediimide-Bithiazole Polymer for n-Type Organic Thermoelectrics. Adv. Mater. 30, 1801898, doi:10.1002/adma.201801898 (2018).

39 Cho, C. et al. Completely Organic Multilayer Thin Film with Thermoelectric Power Factor Rivaling Inorganic Tellurides. Adv. Mater. 27, 2996-3001, doi:10.1002/adma.201405738 (2015).

40 Rivnay, J. et al. Unconventional Face-On Texture and Exceptional In-Plane Order of a High Mobility n-Type Polymer. Adv. Mater. 22, 4359-4363, doi:10.1002/adma.201001202 (2010).

41 Miglbauer, E., Wójcik, P. J. \& Głowacki, E. D. Single-compartment hydrogen peroxide fuel cells with poly(3,4-ethylenedioxythiophene) cathodes. ChemComm 54, 11873-11876, doi:10.1039/C8CC06802J (2018).

42 Savva, A. et al. Influence of Water on the Performance of Organic Electrochemical Transistors. Chem. Mater. 31, 927-937, doi:10.1021/acs.chemmater.8b04335 (2019).

43 Wustoni, S., Savva, A., Sun, R., Bihar, E. \& Inal, S. Enzyme-Free Detection of Glucose with a Hybrid Conductive Gel Electrode. Adv. Mater. Interfaces 0, 1800928, doi:doi:10.1002/admi.201800928.

44 Savva, A., Wustoni, S. \& Inal, S. Ionic-to-electronic coupling efficiency in PEDOT:PSS films operated in aqueous electrolytes. J. Mater. Chem. C, doi:10.1039/C8TC02195C (2018).

45 Bihar, E. et al. A fully inkjet-printed disposable glucose sensor on paper. npj Flexible Electron. 2, 30, doi:10.1038/s41528-018-0044-y (2018). 


\section{Methods}

\section{Materials}

Glucose oxidase (GOx), 3,4-ethylenedioxythiophene (EDOT), hydroxymethyl 3,4ethylenedioxythiophene $(\mathrm{EDOTOH}))$, lithium perchlorate $\left(\mathrm{LiClO}_{4}\right)$ were purchased from SigmaAldrich and used as received. The n-type polymers, P-90 and P-0, were synthesized according to a protocol reported previously. ${ }^{17}$

\section{Fabrication of the OECT and Biofuel Cell}

For the fabrication of OECTs, we patterned the Au interconnects and contacts (located at the the three terminals) on a glass substrate and used a Parylene $\mathrm{C}$ layer to insulate these Au patterns according to an established protocol. ${ }^{43}$ The dimension of the channels was $10 \mu \mathrm{m}$ in length and $100 \mu \mathrm{m}$ in width, whereas the Au electrode used as the gate had an area of $500 \times 500 \mu \mathrm{m}^{2}$. We spincoated the n-type material, P-90, (1000 rpm, $30 \mathrm{~s})$ from a chloroform solution without any annealing or post-processing steps. The film thickness was $40 \mathrm{~nm}$ in the channel and $80 \mathrm{~nm}$ at the gate.

For the fabrication of the biofuel cell, we used $175 \mu \mathrm{m}$ thick flexible Kapton (polyimide) films as substrates. We cut the Kapton with a laser into a specific circular geometry (0.65 $\mathrm{mm}$ in diameter) and subsequently washed in an acetone/isopropyl alcohol and deionized (DI) water baths under sonication for 30 minutes. We then sputtered a 10/100 nm thick $\mathrm{Cr} / \mathrm{Au}$ or Pt layer (when $\mathrm{Pt}$ was used as a cathode) on top of the substrates. As a final step, the electrodes were cleaned in acetone and sonicated for 30 minutes, followed by a rinse and soak in DI water under sonication for the same amount of time. For the bioanode, we spin-coated P-90 solution (10-15 $\mu \mathrm{L}$ aliquots) in chloroform on top of the active area of the Au coated flexible polyimide substrate $\left(0.33 \mathrm{~cm}^{2}\right)$ by a two-step coating (350 rpm.10s $\mathrm{s}^{-1}$ followed by $1000 \mathrm{rpm} .30 \mathrm{~s}^{-1}$ ). Upon natural drying of the film, we 
drop-casted the GOx solution in phosphate-buffered saline (PBS) $\left(10 \mathrm{mg} \cdot \mathrm{mL}^{-1}\right)$ on top of the electrode (i.e., immobilization of GOx through enzyme adsorption) and left to dry under ambient conditions for a minimum of 30 minutes. For the cathode, we first electrochemically cleaned the Au coated polyimide electrode in an aqueous solution of $\mathrm{H}_{2} \mathrm{SO}_{4}(10 \mathrm{mM})$ via cyclic voltammetry (CV, from $-0.4 \mathrm{~V}$ to $1.2 \mathrm{~V} v s$. $\mathrm{Ag} / \mathrm{AgCl})$ for 10 cycles. Then, electropolymerization of $\mathrm{p}$ (EDOTco-EDOTOH) on the Au electrode was performed potentiostatically at $1 \mathrm{~V}$ for $10 \mathrm{~min}$ in an aqueous solution containing EDOT $(10 \mathrm{mM})$, EDOTOH $(10 \mathrm{mM})$ and $\mathrm{LiClO}_{4}(100 \mathrm{mM})$ using a potentiostat-galvanostat (Autolab PGSTAT128N, MetroOhm). Subsequently, the substrate was rinsed with DI water and dried with $\mathrm{N}_{2}$ gas. Glass vials (Ossila, C20052) were used for the membrane-free cells where the inter-electrode gap distance was $\sim 0.5 \mathrm{~cm}$. For the case of the membrane cell, a cationic exchange membrane separated the anode and the cathode at a distance of $\sim 1.5 \mathrm{~cm}$ (Nafion 117, Sigma Aldrich) to maintain electroneutrality. Our custom-built EFC was made from poly(methyl methacrylate) (PMMA) and could accommodate $2 \mathrm{~mL}$ of the solution on each side.

\section{Electrochemical characterization}

All characterizations were performed in PBS ( $\mathrm{pH}$ 7.4). The cyclic voltammograms of the films were recorded using a potentiostat-galvanostat (Autolab, PGSTAT128N, MetroOhm) with an $\mathrm{Ag} / \mathrm{AgCl}$ reference electrode (3 $\mathrm{M} \mathrm{KCl}$, ALS co. Ltd.) and a Pt counter electrode (RE-1B, ALS co. Ltd.) in $\mathrm{N}_{2}$ or $\mathrm{O}_{2}$ saturated environments (e.g. in PBS and air) as well as in the absence or presence of glucose. The working electrode was an electropolymerized p(EDOT-co-EDOTOH) film or a P-90 film cast on top of an Au coated substrate. As for the investigation of the $\mathrm{O}_{2}$ reduction capability of the biofuel cell cathode p(EDOT-co-EDOTOH), we used a rotating disk electrode system (RDE710 Rotating Electrode, Gamry Instruments). The film was 
electropolymerized on the glassy carbon electrode following the same procedure explained above. The RDE system was coupled to a channel MultiEmStat3+ (Palmsens) potentiostat and voltammograms were obtained by varying the electrode rotation rate and the potential applied at a scan rate of $5 \mathrm{mV} \cdot \mathrm{s}^{-1}$. All experiments were performed in PBS using a Pt wire as the counter electrode and $\mathrm{Ag} / \mathrm{AgCl}(3 \mathrm{M} \mathrm{KCl})$ as the reference electrode.

\section{Quartz crystal microbalance with dissipation monitoring (QCM-D)}

We conducted QCM-D measurements using a Q-sense analyzer (QE401, Biolin Scientific AB, Sweden) with $\mathrm{Cr} / \mathrm{Au}$ coated quartz crystals before (used as reference) and after coating with the polymer films. After stabilizing the film in the buffer solution (PBS), we introduced the GOx solution $\left(10 \mathrm{mg} \cdot \mathrm{mL}^{-1}\right)$ into the chamber. The frequency $(\Delta f)$ and dissipation $(\Delta D)$ signals were recorded until stabilized, followed by a PBS rinsing step to allow loosely bound proteins to desorb. The measured shifts in the frequency of the sensors were converted into changes in mass $(\Delta m)$ using the Sauerbrey equation:

$$
\Delta m=\frac{-17.7}{\mathrm{n} * \Delta \mathrm{f}}
$$

where $n$ is the number of the selected overtone for the quantification of the mass and -17.7 is a constant determined on the resonant frequency, active area, density and shear modulus of the crystal. $^{44}$

\section{In situ UV-VIS-NIR and Raman Spectroscopy}

P-90 film was coated on an ITO substrate following the spin-coating procedure described above. We used an Ocean Optics QE Pro Scientific grade spectrometer (185-1050 nm) to record the UVVIS-NIR spectra of the films. For the spectroelectrochemistry measurements, a Keithley 2606A source measure unit was coupled to the sample holder which contains the film submerged in the 
electrolyte. When required, we appled a bias between an $\mathrm{Ag} / \mathrm{AgCl}$ electrode and the P-90 film addressed as the working electrode, in the absence or presence of the enzymatic reaction.

For the in situ Raman spectroelectrochemical investigation, P-90 films coated on Au substrates were exposed to a drop of PBS into which an $\mathrm{Ag} / \mathrm{AgCl}$ electrode was immersed. The electrochemical area $\left(1 \mathrm{~cm}^{2}\right)$ was defined as a square aperture in a Parafilm medium where $5 \mu \mathrm{L}$ of the solution (PBS, with or without glucose) was placed. The bias was applied using a Keithley 2600B source meter. The near-resonance Raman spectra were measured using a Witec alpha Raman spectrometer in backscattering configuration with a linearly polarized excitation of $\mathrm{He}-\mathrm{Ne}$ laser of wavelength $632.8 \mathrm{~nm}$, and a power level $<500 \mu \mathrm{W}$ to avoid photo-thermal effects. The dispersion gratings used, $600 \mathrm{~g} \cdot \mathrm{mm}^{-1}$, allowed to collect a spectral range up to $2700 \mathrm{~cm}^{-1}$, covering the spectral area of interest completely. A Zeiss $63 x, N A=1$, water immersion objective focused on the polymer surface was used to excite the sample and collect the Raman signal. For each condition, we mapped the sample with 10 points for $2 \mathrm{sec}$ to average out local statistical fluctuation, thus defining the representative spectrum. The Raman spectra presented here were obtained by removing the bias-dependent baseline, described by a $4^{\text {th }}$ order polynome, and after the normalization to the Я-mode peak intensity of the bithiophene units $\left(1457 \mathrm{~cm}^{-1}\right)$. Note that the baseline treatment enabled tracking the bias-triggered evolution of the polaronic excitation in the copolymer backbone responsible for strong light absorption, emission, and Raman scattering. Using this procedure, we could compare the relative intensities and shifts of the main peaks recorded for different samples and at various biasing conditions in the absence and presence of enzymatic reaction.

\section{Scanning Electron Microscopy (SEM)}


SEM images were obtained using Nova Nano SEM. P-90 films were deposited on glass coverslips and coated with a $5 \mathrm{~nm}$ of iridium before imaging. For the wet conditions, the films were immersed in deionized water overnight to ensure that they swell. The samples were then frozen using liquid $\mathrm{N}_{2}$ and sublimated inside the cryo-SEM chamber.

\section{Chronoamperometric sensing measurements}

To evaluate the sensor performance, we drop-casted GOx in PBS (10 mg.mL $\left.\mathrm{mL}^{-1}\right)$ on the device active area (channel and gate) and left for 30 minutes to physically adsorb. The active area was defined by the dimensions of the glass well (diameter of $4 \mathrm{~mm}$ ) immobilized on top of the device to confine the electrolyte solution. We recorded the current-voltage characteristics of the devices using a Keithley 2602A dual source meter. We monitored, in real-time, changes in the drain current of the OECT at a constant source-drain voltage and a gate voltage. After a steady baseline was obtained for the drain current (current at zero analyte concentration), we monitored the real-time changes in response to subsequent additions of increasing concentrations of glucose into the electrolyte. For all experiments, the electrolyte volume was kept at $40 \mu \mathrm{L}$. For an accurate comparison between different devices, we normalized the response of the device to glucose as follows:

$$
\mathrm{NR}=\left|\frac{I_{\mathrm{D}}-I_{0}}{I_{0}}\right|
$$

where $I_{\mathrm{D}}$ and $I_{0}$ are the current output at a given analyte concentration and zero analyte concentration, respectively. Solutions of enzyme and glucose were stored at $4^{\circ} \mathrm{C}$. For the control experiments involving the non-catalytic enzyme, we heated the GOx solution $\left(100^{\circ} \mathrm{C}\right.$ for 30 minutes) to obtain the denatured form of the protein. Chronoamperometric sensing measurements were then performed with the OECTs functionalized with this enzyme. For the biosensor selectivity assay, we measured the current response of the OECT to glucose, lactate, ascorbic acid, 
and uric acid at physiologically relevant concentrations, ${ }^{45}$ both in the absence and presence of GOx.

\section{Biofuel cell characterization}

We electrochemically characterized the half-cells and biofuel cells using a MultiEmStat3+ (Palmsens) potentiostat. For half-cell characterization, cyclic voltammograms at ambient temperature were recorded in a three-electrode set up using an $\mathrm{Ag} / \mathrm{AgCl}$ reference electrode and a Pt foil counter electrode. We coated the P-90 film and electropolymerized p(EDOT-co-EDOTOH) on $\mathrm{Au}$ sputtered substrates for anode and cathode characterization, respectively. For the measurements performed under inert atmosphere, the system was degassed in a closed chamber for at least 15 minutes in $\mathrm{N}_{2}$ prior experimentation.

For the EFC characterization, we supplied various glucose concentrations to the biofuel cell using a peristaltic syringe pump (Ossila, L2003S1) and recorded the open circuit voltage (OCV) of the cell throughout 30-minute intervals. We obtained all the power curves by measuring the cell voltage across a variable load resistor $(1 \mathrm{k} \Omega-10 \mathrm{M} \Omega)$. Once a resistor of fixed value was applied, each point was measured after 30 minutes of stabilization period to ensure a stable voltage output. Using Ohm's law, we calculated current and power densities normalized by the geometrical surface area of the electrodes. For estimation of power densities at extended potentials, we recorded linear sweep voltammetry (LSV) curves (up to 1.2.V) at a scan rate of $5 \mathrm{mV} . \mathrm{s}^{-1}$ To obtain the inflection points and corresponding power retentions, we calculated the first derivative of the LSV plot. The total volume of the solution used for each measurement was $1 \mathrm{~mL}$. The operating temperature of the $\mathrm{EFC}$ was $25^{\circ} \mathrm{C}$.

\section{Stability of the biofuel cell}


We attested the stability of the biofuel cells by measuring the change in their OCV and the power density values over time. We used the syringe pump with a rate of $150 \mu \mathrm{L} \cdot \mathrm{s}^{-1}$ to feed the cell. We investigated the effect of enzyme replenishment as well as of enzyme encapsulation (using a Nafion 117 film) on device stability. The electrodes were stored in ambient conditions after each measurement.

\section{Hydrogen peroxide detection}

For the detection of hydrogen peroxide $\left(\mathrm{H}_{2} \mathrm{O}_{2}\right)$, we collected aliquots $(0.1 \mathrm{~mL})$ of the EFC electrolyte during its operation with a disposable syringe (Terumo). We then used a peroxide assay kit (Sigma Aldrich) to determine the concentration of $\mathrm{H}_{2} \mathrm{O}_{2}$ in these aliquots. The assay utilizes the chromogenic $\mathrm{Fe}^{3+}$-xylenol orange reaction, in which a purple complex is formed when $\mathrm{Fe}^{2+}$ is oxidized to $\mathrm{Fe}^{3+}$ by the $\mathrm{H}_{2} \mathrm{O}_{2}$ present in the sample, generating a colorimetric result $(585 \mathrm{~nm})$. A spectrophotometer (Promega) was used to measure the absorbance intensity, which scales with $\mathrm{H}_{2} \mathrm{O}_{2}$ concentration.

\section{Experiments with human saliva samples}

For the experiments using saliva, we collected the saliva of healthy volunteers after fasting (12 hours). We determined the glucose concentration in these samples through the use of a commercial Glucose Assay Kit (GAGO-20, Sigma Aldrich) and a spectrophotometer (Promega). To mimic physiological variations of glucose in saliva, we added different concentrations of glucose to this sample. All protocols and procedures involving human saliva were approved by the KAUST Institutional Biosafety and Bioethics Committee (IBEC). The volunteers provided signed consent to participate in the study. Saliva samples were collected and frozen at $-20^{\circ} \mathrm{C}$. Fresh solutions were made for each new measurement. 


\section{Reference Methods}

43 Wustoni, S., Savva, A., Sun, R., Bihar, E. \& Inal, S. Enzyme-Free Detection of Glucose with a Hybrid Conductive Gel Electrode. Adv. Mater. Interfaces 0, 1800928, doi:doi:10.1002/admi.201800928.

44 Savva, A., Wustoni, S. \& Inal, S. Ionic-to-electronic coupling efficiency in PEDOT:PSS films operated in aqueous electrolytes. J. Mater. Chem. C, doi:10.1039/C8TC02195C (2018).

45 Bihar, E. et al. A fully inkjet-printed disposable glucose sensor on paper. npj Flexible Electron. 2, 30, doi:10.1038/s41528-018-0044-y (2018). 


\section{Acknowledgments}

The authors thank Dr. Eloise Bihar (KAUST) for her guidance during experiments with saliva samples. The authors would also like to thank Jokubas Surgailis, José Ilton de Oliveira Filho, Luis Ballesteros Ospina and Tania Cecilia-Hidalgo (KAUST) for spontaneous help. Funding: D.O., I.M. and S.I. gratefully acknowledge financial support from the KAUST Office of Sponsored Research (OSR) under Award No. OSR-2016-CRG5-3003. G.N. and S.I. acknowledge support from KAUST Sensors Initiative OSR Award No. REP/1/2719.

Competing Interests: A U.S. provisional application (no. 62/770934) related to this work was filed by S.I. and D.O.

Author contributions: S.I. conceived the research, designed the experiments, and supervised the work. D.O, G.N. and A.S performed the OECT and biofuel cell experiments. D.O. fabricated the devices and performed the UV-VIS-NIR studies. S.W. designed the electropolymerization experiments. X.C, I.P.M., and I.M. provided the n-type materials. P.D.C. and T.P. helped with the biofuel cell experiments. A.S. and D.O. performed the QCM-D experiments. A.G. performed and E.D.F supervised the Raman spectroscopy measurements. D.O. and G.N wrote the manuscript with S.I. All authors were involved in the discussion and participated in manuscript input. Figure 1A and Figure 4A were produced by Heno Wang, scientific illustrator at KAUST.

Data and materials availability: All data needed to evaluate the conclusions in the paper are present in the paper and/or the Supplementary Materials. Additional data related to this paper may be requested from the authors. 